\title{
The role of connectivity and noise in the emergence of spontaneous activity in cultured neuronal networks
}

\author{
Javier G. Orlandi ${ }^{\dagger}$, Enrique Alvarez-Lacalle ${ }^{\ddagger}$, Sara Teller ${ }^{\dagger}$, Jaume Casademunt ${ }^{\dagger}$, and Jordi Soriano ${ }^{\dagger}$ \\ $†$ Departament d'ECM, Facultat de Física, Universitat de Barcelona. \\ Martí i Franqués 1, 08028 Barcelona, Spain \\ \$Departament Física Aplicada, EETAC. Universitat Politècnica de Catalunya BarcelonaTech. \\ Esteve Terrades 5, 08860 Castelldefels, Spain \\ Email: orlandi@ecm.ub.es
}

\begin{abstract}
Cultured neurons in vitro quickly connect to one another to establish a spontaneously active network within a week. The resulting neuronal network is characterized by a combination of excitatory and inhibitory integrate-and-fire units coupled through synaptic connections, and that interact in a highly nonlinear manner. The nonlinear behavior emerges from the dynamics of both the neurons' membrane potential and synaptic transmission, together with intrinsic biological noise. These ingredients give rise to a rich repertoire of phenomena that are still poorly understood, including periodic spontaneous activity, avalanches, propagation of activity fronts, and synchronization. Here we describe some experimental results on spontaneous activity in cultures, and elaborate on theoretical models that describe its initiation and maintenance.
\end{abstract}

\section{Introduction}

Neuronal activity in the mammalian brain is a prominent example of how individual units (neurons) interact to achieve a larger goal or function. The processing of external information and the interaction between different brain circuits gives rise to a wide range of dynamical processes that are crucial for learning, memory, and behavior. The interaction between neurons is based on the ability of one neuron to evoke (excitatory neurons) or inhibit (inhibitory neurons) the generation of action potentials in the neurons that they connect to, generally via synapses.

Neuronal interactions are embedded in such an intricate network of connections that relating a particular brain process or function to the underlying neuronal activity is not only a difficult task, but also one of the major challenges of modern neuroscience [1]. A first step in the quest for understanding the interplay activity-connectivity-function consists in the identification of the mechanisms behind spontaneous activity. Rhythmic spontaneous episodes of activity are widespread in neuronal tissues, for instance in the form of brain rhythms [2], retinal waves or spinal cord bursting. Spontaneous activity plays a pivotal role in diverse brain tasks, and is crucial for the correct formation, survival and refinement of neuronal circuits.

Spontaneous activity is not only limited to naturally-

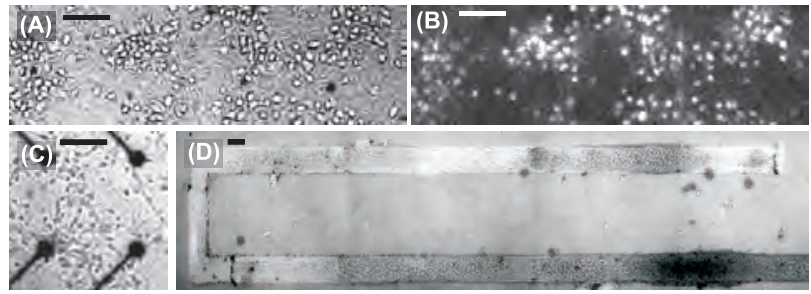

Figure 1: Neuronal cultures. (A)-(B) Neurons grown on a glass cover slip, showing a bright field (left) and calcium fluorescence (right) images. Circular objects in (A) and bright spots in (B) are neurons. (C) Culture on a MultiElectrode Arrays system. (D) One-dimensional neuronal culture on a pre-patterned line $170 \mu \mathrm{m}$ wide and $12 \mathrm{~mm}$ long. Scale bar is $100 \mu \mathrm{m}$.

formed neuronal tissues. Brain slices are also spontaneously active and even retain some of the dynamical traits of the original tissue. Furthermore, the dissociation, plating and culture of circuits as diverse as cortical or hippocampal brain areas, retina or spinal cord, lead to networks that selforganize, grow and mature to constitute a spontaneously active network [3].

The robust presence of spontaneous activity in such a dramatically diverse neuronal circuits hints at the presence of general mechanisms - in both neuronal dynamics and connectivity - that initiate and control it. Based on experimental observations in neuronal cultures, here we discuss on these mechanisms and their role in shaping spontaneous activity in neuronal networks.

\section{Experiments}

Neuronal cultures (Fig. 1) have become one of the most attractive in vitro systems to study spontaneous activity [3]. Cultures are typically prepared from specific regions of embryonic rat brains, and after dissociation and plating. Neurons connect within a day, and show rich spontaneous activity as early as day in vitro (DIV) 5-6 [4].

Activity in cultures is typically monitored through Calcium Fluorescence Imaging (Fig. 1A-B), which can simultaneously record thousands of neurons in large areas at $\sim 10$ 


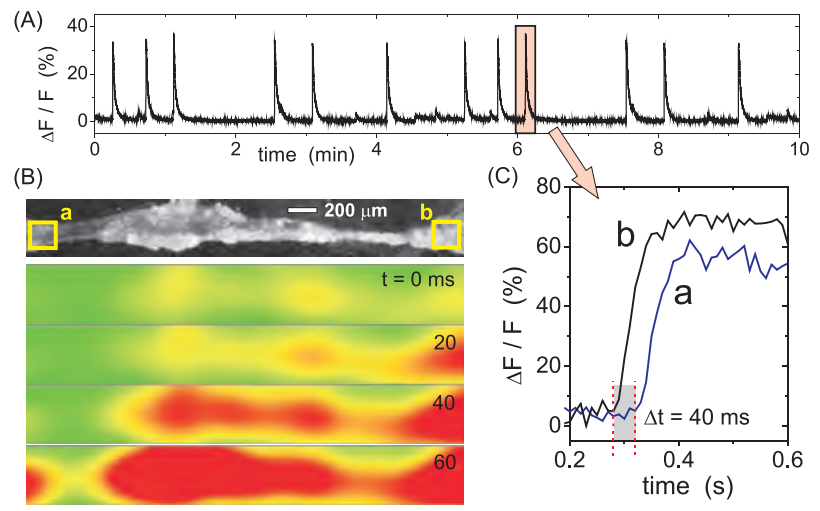

Figure 2: Activity in unidimensional neuronal cultures. (A) Spontaneous activity recorded through calcium fluorescence imaging at 100 frames/s, averaged over the network. Fluorescence peaks are bursting episodes, each of them corresponding to the generation and propagation of an activity front. (B) Fluorescence image of a region of the culture depicting two Regions of Interest (ROIs) and the advance of a propagating front, which crosses the field of view from right to left at $70 \mathrm{~mm} / \mathrm{s}$. Color coding is proportional to fluorescence amplitude. (C) Determination of the propagation velocity within a burst by calculating the time-delay in neuronal activation between two ROIs, $a$ and $b$. Fluorescence amplitude is shown normalized respect to the baseline $F_{0}$, with $\Delta F=F-F_{0}$.

ms resolution, or through Multi-Electrode Arrays (MEAs) (Fig. 1C) [3], which deliver high, $\sim \mu$ s resolution but with a limited number of recording sites, on the order of 100. Spontaneous activity in cultures is characterized by bursting episodes of collective neuronal activation combined with quieter inter-bursts intervals (Fig. 2A).

One of the simplest experimental systems to study the initiation and propagation of spontaneous activity is unidimensional neuronal cultures. They are obtained by setting up a dimension of the culture much smaller than the characteristic diameter of the neuron's dendritic tree (around $300 \mu \mathrm{m})$. Unidimensional cultures were introduced in Refs. $[5,6]$ by chemically constraining the growth of the neurons to a narrow band of about $200 \mu \mathrm{m}$ wide (Fig. 1D). As an example, we consider here an alternative approach based on the confinement of neurons and connections in topographical molds, with neurons growing only in a long, narrow valley carved on a polymer (Fig. 2B).

Spontaneous activity in 1D cultures is characterized by pulses (bursts) of neuronal activity that initiate in localized zones along the line, termed burst initiation zones. The velocity of a propagating pulse (Fig. $2 \mathrm{~B}$ ) is determined by measuring the difference in activation times along consecutive regions in the line, as shown in Fig. 2C.

The experiments described in Refs. [5, 6] show that activity fronts propagate at constant velocity, sequentially activating the neurons in their path. Axons grow roughly par- allel to the line and therefore neurons easily connect to their neighbors, forming a chain-like network that extends with equal probability towards both ends of the line. A detailed analysis of the properties of initiation and propagation of activity shows that, on one hand, the velocity of the activity front predominantly depends on the connectivity properties of the network [5]. For instance, networks with both excitatory and inhibitory neurons provide velocities around $60 \mathrm{~mm} / \mathrm{s}$, which almost double when inhibition is blocked. On the other hand, the existence of localized burst initiation zones is ascribed to both a stronger connectivity and recurrent network activity at the vicinity of the initiation zone [6]. These results evidence that the initiation and propagation of spontaneous activity depends on a subtle interplay between neuronal dynamics and connectivity.

The investigation of activity-initiation mechanisms and burst propagation in the more complex bi-dimensional neuronal cultures is difficult since one has to access large areas, and with both high temporal and spatial resolution. Therefore, most of the studies have used MEAs for its high temporal resolution. In the quest for understanding burst initiation, some studies identified a subset of neurons termed leaders that were always the first to ignite, and that induced the firing of the entire neuronal population. It was also observed that activity in this subset increased exponentially in a short time window prior to the burst [7]. The authors hypothesized that such a fast recruitment required the leader neurons to be present all over the network, possibly forming a subnetwork of highly connected neurons $[8,9]$.

Since connectivity in cultures increases with neuronal density [4], it may be possible that neuronal aggregates (such as the ones observed in Fig. 1B) or strong fluctuations in the spatial distribution of neurons give rise to highly connected regions that are foci of activity. Hence, given the poor spatial resolution of MEAs, it may occur that leader neurons actually correspond to regions with specific topological properties that favor initiation, rather than a special kind of cells.

\section{Modeling neuronal cultures dynamics}

One of the key findings from the experiments in unidimensional cultures $[5,10]$ is the identification of two propagation regimes. A first one corresponds to a slow propagation of the activity front that is stable only in particular conditions. This regime is transitory and lasts a few milliseconds. The second regime corresponds to the stable, fast propagating front (such as the one of Fig. 2B) that advances along the line at constant velocity.

The theoretical and numerical analysis [10] behind these experiments showed that, to reliable reproduce the experimental observation in neuronal cultures, one has to introduce more complex scenarios that go beyond simple Integrate-and-fire models. Indeed, an appropriate neuron model must include first a slow variable that mimics the presence of slow $\mathrm{K}^{+}$channels in the soma. Second, it must 
include spike frequency adaptation in order to explain the transient regime that precedes the fast propagation. The set of equations that include these ingredients while keeping the minimum number of components give rise to the twodimensional Izhikevich model [11],

$$
\begin{aligned}
\tau_{c} \dot{v} & =k\left(v-v_{r}\right)\left(v-v_{t}\right)-u+I+\eta, \\
\tau_{a} \dot{u} & =b\left(v-v_{r}\right)-u, \\
\text { if } v \geq v_{p} & \text { then } \quad v \leftarrow v_{c}, u \leftarrow u+d .
\end{aligned}
$$

Here $v$ is the membrane potential, $v_{r}$ the resting potential, $v_{t}$ the threshold potential, $u$ is the slow adaptation variable with time constant $b, I$ the inputs received and $\eta$ a noise term. In the model, a spike is generated when the membrane potential reaches a value $v_{p}$. Then, $v$ is reset to a new value $v_{c}$ and $u$ is increased by a factor $d$. The quantities $c$ and $d$ are neuron-specific parameters, allowing the incorporation of different neuronal types within the same model.

In addition to the spiking model, another key ingredient that is needed to account for all the observations is the dynamics of neuronal connections. Here we consider the connections between two neurons $i$ and $j$ as chemical synapses with short-term depression [12]. An action potential generated by neuron $i$ travels down the axon and releases neurotransmitters in the synaptic cleft. These neurotransmitters bind to specific receptors in the presynaptic terminal of neuron $j$, generating a current in its soma. Synaptic depression takes into account the finite number of available neurotransmitters and their dynamics. The whole process can be modeled $[10,12]$ as

$$
\begin{aligned}
E_{i}\left(t, t_{m}\right) & =g D_{i}\left(t_{m}\right) \exp \left(-\frac{t-t_{m}}{\tau}\right) \theta\left(t-t_{m}\right) \\
\dot{D} & =\frac{1}{\tau_{D}}(1-D)-(1-\beta) D \delta\left(t-t_{m}\right),
\end{aligned}
$$

where $E_{i}\left(t, t_{m}\right)$ is the current generated at neuron $j$ by a spike from neuron $i$ at time $t=t_{m}, g$ is the strength of the synapse and $D(t)$ is the synaptic depression. The current decays exponentially with a time constant $\tau_{m}$. Each spike from neuron $i$ causes the variable $D$ to decrease to $\beta D$, with $\beta<1$, recovering with a time scale $\tau_{D}$.

In the absence of stimuli, neurons in culture fire spontaneously with a characteristic frequency that follows Poisson statistics. Although the source of spontaneous activity is still unclear, some recent studies $[13,14]$ hint at miniature post-synaptic currents generated by the spontaneous release of neurotransmitter's vesicles at the synapses. This minis can be considered as a shot noise, i.e. a Poisson process acting at each synapse and that generates post-synaptic currents like Eq. (4), but of smaller amplitude.

Finally, although the dynamic behavior of neurons and connections is essential, another key element needed to fully understand the collective dynamics in neuronal cultures is the structure of the network itself. The rich activity observed in any neuronal tissue arises from the interplay between dynamics and wiring architecture. In the
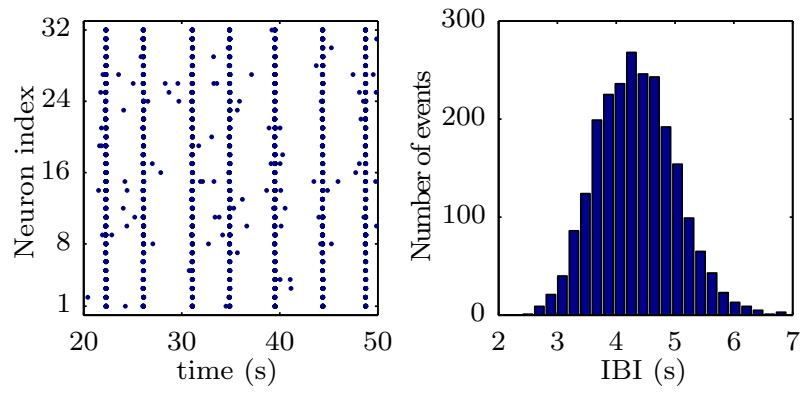

Figure 3: Burst dynamics in a simulated culture. Left, raster plot showing the spike times of 32 randomly selected neurons in the culture. Spike times are marked with blue dots along the $\mathrm{x}$ axis. Bursts appear as bands of collective activity. Right: distribution of inter burst intervals (IBIs), illustrating its high periodicity.

unidimensional case the topological structure is straightforward and one can assume a connectivity probability between neurons that decays with distance. In two- and threedimensional networks, however, the increased dimensionality allows for more complex connectivity patterns. In terms of modeling, one then has to consider different network structures, such as random, small-world or scale-free topologies, or even a combination of them.

\subsection{Discussion and conclusions}

With the above considerations, the global bursts of activity observed in cultures (e.g. Fig. 2A) seem to emerge from a stochastic process that can be mapped to a problem of percolation $[15,16]$. When a critical fraction of neurons become active at the same time, they can excite all their neighbors and in turn, through an iterative process, the entire network. One would expect that, due to the stochastic origin of these bursting events, the inter burst interval (IBI) would follow an exponential-like distribution. The combination of many Poisson processes (single neuron firings) should give rise to an IBI distribution peaked around zero time and with a fast decay. However, this analysis contradicts the experimental evidence that bursting is remarkably periodic, with an IBI distribution that is Gaussian rather than exponential. The average IBI falls in the range $1-100$ $\mathrm{s}$, depending on the experimental conditions.

The observation that neuronal cultures have a characteristic time scale of seconds, while all the system's internal variables are of the order of ms, can only be explained by the presence of synaptic depression in the system [14]. The time associated to vesicle recycling in the system is of the order of seconds, and the combination of this time scale with the stochastic origin of activity gives rise to the periodicity of the bursts, as illustrated in Fig. 3 for numerical simulations. All the synapses in the system are depleted after a burst of activity, and the network needs a long time to recover. Another burst can emerge only when the synapses 
have recovered up to a given threshold.

The picture of burst initiation becomes less clear when more detailed topological properties are included in systems beyond unidimensional cultures. Studies using MEAs in two dimensional cultures have not revealed any clear pattern of activity, and several studies have theorized about the driving mechanisms of bursting behavior. From the concept of leader neurons [8,9] to the hypothesis of different network structures and neuronal subtypes [17]. It was also suggested, based on both experiments and theoretical models, that activity may be described as neuronal avalanches with distributions that resemble those observed in Self-Organized Criticality [18].

In conclusion, the initiation and propagation of spontaneous activity is a challenging problem that has not been fully addressed yet. At an experimental level, one needs to improve activity visualization techniques in large, bidimensional cultures in order to accurately resolve propagating fronts. One also needs to devise strategies to dictate and map out network connectivity in a control manner, a fundamental step towards the exploration of the interplay between wiring architecture and network activity. From a theoretical and numerical points of view, there are still several questions open on the isolation of the fundamental ingredients at the dynamical and and topological levels, particularly the incorporation of realistic, experimentally measured connectivity maps and plasticity.

\section{References}

[1] E. Bullmore and O. Sporns, "Complex brain networks: graph theoretical analysis of structural and functional systems.," Nat Rev Neurosci, vol. 10, pp. 186-198, Mar. 2009.

[2] G. Buzsáki and A. Draguhn, "Neuronal oscillations in cortical networks.," Science, vol. 304, pp. 1926-1929, June 2004.

[3] J.-P. Eckmann, O. Feinerman, L. Gruendlinger, E. Moses, J. Soriano, and T. Tlusty, "The physics of living neural networks," Physics Reports, vol. 449, pp. 54-76, Sept. 2007.

[4] J. Soriano, M. Rodríguez Martínez, T. Tlusty, and E. Moses, "Development of input connections in neural cultures.," Proceedings of the National Academy of Sciences, vol. 105, pp. 13758-13763, Sept. 2008.

[5] O. Feinerman, M. Segal, and E. Moses, "Signal propagation along unidimensional neuronal networks.," Journal of Neurophysiology, vol. 94, pp. 3406-3416, Nov. 2005.

[6] O. Feinerman, M. Segal, and E. Moses, "Identification and dynamics of spontaneous burst initiation zones in unidimensional neuronal cultures.," Journal of Neurophysiology, vol. 97, pp. 2937-2948, Apr. 2007.

[7] D. Eytan and S. Marom, "Dynamics and effective topology underlying synchronization in networks of cortical neurons.," Journal of Neuroscience, vol. 26, pp. 8465-8476, Aug. 2006.

[8] J.-P. Eckmann, S. Jacobi, S. Marom, E. Moses, and C. Zbinden, "Leader neurons in population bursts of 2D living neural networks," New J. Phys., vol. 10, p. 5011, Jan. 2008.

[9] J.-P. Eckmann, E. Moses, O. Stetter, T. Tlusty, and C. Zbinden, "Leaders of neuronal cultures in a quorum percolation model.," Front. Comput. Neurosci., vol. 4, 2010.

[10] E. Alvarez-Lacalle and E. Moses, "Slow and fast pulses in 1-D cultures of excitatory neurons.," J Comput Neurosci, vol. 26, pp. 475-493, June 2009.

[11] E. M. Izhikevich, "Simple model of spiking neurons.," IEEE Trans Neural Netw, vol. 14, no. 6, pp. 1569-1572, 2003.

[12] M. Tsodyks, A. Uziel, and H. Markram, "Synchrony generation in recurrent networks with frequencydependent synapses.," Journal of Neuroscience, vol. 20, p. RC50, Jan. 2000.

[13] D. Cohen and M. Segal, "Homeostatic presynaptic suppression of neuronal network bursts.," Journal of Neurophysiology, vol. 101, pp. 2077-2088, Apr. 2009.

[14] D. Cohen and M. Segal, "Network bursts in hippocampal microcultures are terminated by exhaustion of vesicle pools.," Journal of Neurophysiology, vol. 106, pp. 2314-2321, Nov. 2011.

[15] T. Tlusty and J.-P. Eckmann, "Remarks on bootstrap percolation in metric networks," J. Phys. A: Math. Theor., vol. 42, p. 5004, May 2009.

[16] O. Cohen, A. Keselman, E. Moses, M. Rodríguez Martínez, J. Soriano, and T. Tlusty, "Quorum percolation in living neural networks," Europhysics Letters, vol. 89, p. 18008, Jan. 2010.

[17] I. Baruchi, V. Volman, N. Raichman, M. Shein, and E. Ben-Jacob, "The emergence and properties of mutual synchronization in in vitro coupled cortical networks.," Eur. J. Neurosci., vol. 28, pp. 1825-1835, Nov. 2008.

[18] A. Levina, J. M. Herrmann, and T. Geisel, "Dynamical synapses causing self-organized criticality in neural networks," Nature Physics, vol. 3, pp. 857-860, Dec. 2007. 